\title{
Conocimientos en Salud Sexual y Reproductiva en Estudiantes de Enfermería de una Universidad de Barranquilla (Atl, Col)
}

\section{Knowledge in Sexual and Reproductive Health in Nursing Students of University of Barranquilla (Atl, Col)}

Cano Ibarra SM, Guerra Ramírez M, Montenegro Rivera C. ${ }^{1}$

'Universidad Simón Bolívar, Barranquilla, Colombia.

Resumen - Los avances a nivel mundial en relación a los de derechos humanos, han permitido que los adolescentes hayan logrado un amplio reconocimiento de sus derechos sexuales y reproductivos. Éstos, en ocasiones han sido violentados por aspectos de su sexualidad, trayendo consecuencias y prejuicios sobre su desarrollo y capacidad para tomar decisiones.

Objetivo. Determinar que conocimientos sobre salud sexual y reproductiva poseen las estudiantes del primer semestre de Enfermería de una Universidad de Barranquilla.

Materiales y métodos. Estudio cuali-cuantitativo, descriptivo, no experimental, de corte transversal. Se estudió una población de 90 estudiantes de primer semestre de Enfermería de una universidad de Barranquilla, Colombia. Se utilizó como técnica la encuesta conformado por veintiocho (28) ítems con respuestas de selección simple, validado por juicio de expertos.

Resultados. El $84,45 \%$ de los participantes fueron mujeres, con edades 20 años. El 82,22\% provenía de la zona urbana, con inicio de vida sexual antes de los 19 años. Aunque el 100\% poseía información previa sobre salud sexual y reproductiva, no tenían el comportamiento adecuado en relación a este tema. El 96,67\% de los encuestados señalan que la universidad tenga un programa de salud sexual y reproductiva.

Conclusión. La población universitaria refleja que no poseen los conocimientos suficientes sobre sus derechos sexuales y reproductivos. Por lo que se planea organizar un programa con el apoyo interdisciplinario de otros profesionales como medicina y psicología, permitiendo preparar al adolescente en esta importante etapa de su vida, contribuyendo a la disminución de ETS, y embarazos no deseados.

Palabras claves: salud sexual, salud reproductiva, Conocimientos, Actitudes y Práctica en Salud (DeCS).

Background: Advances worldwide in relation to human rights, have allowed teenagers have gained wide recognition of their sexual and reproductive rights. They sometimes have been violated by aspects of their sexuality, bringing consequences and prejudices about their development and ability to make decisions.

Objective. Determine that knowledge about sexual and reproductive health have the students of the first semester of Nursing of University of Barranquilla.

Methods. Qualitative and quantitative study, descriptive, not experimental, cross-sectional. A population of 90 fresh men nursing at a university in Barranquilla, Colombia was studied. The survey comprised of twenty (28) items with simple screening responses validated by

Citar: Cano Ibarra SM, Guerra Ramírez M, Montenegro Rivera C. Conocimientos en salud sexual y reproductiva en estudiantes de enfermería de una universidad de Barranquilla (Atl, Col). Cienc. innov. salud. 2015; 3 (2):17 - 22. Recibido: Mar. 20 de 2015 / Modificado: may. 30 de 2015 / Aceptado: Jul. 01 de 2015. expert judgment was used as technique.

Results. The $84.45 \%$ of the participants were women, aged $\leq 20$ years. The $82.22 \%$ came from the urban area, with first sexual inter course before age 19. Although $100 \%$ had prior information about sexual and reproductive health, they did not have the appropriate behavior in relation to this issue. The $96.67 \%$ of respondents said that the university has a program of sexual and reproductive health.

Conclusion. The student population reflects that do not have sufficient knowledge about their sexual and reproductive rights. As plans to organize an interdisciplinary program with the support of other professionals such as medicine and psychology, allowing the teenager to prepare for this important stage of his life, contributing to the decline of STDs and unwanted pregnancies.

Keywords: Sexual Health, Reproductive Health, Health Knowledge, Attitudes, Practice (DeCS).

\section{INTRODUCCIÓN}

En el desarrollo integral de los seres humanos la sexualidad es punto fundamental para su bienestar, al igual que la Salud Sexual y Reproductiva, definiéndose como el completo bienestar físico, mental y social, no solo en la ausencia de enfermedades sino en el plano sexual y reproductivo $(1,2)$.

De esta manera, el ser humano tiene el deber ejercer y exigir sus derechos sexuales y reproductivos, permitiendo de esta forma gozar su sexualidad, reproducirse y regular su fertilidad. Producto del desconocimiento que poseen algunos adolescentes sobre este tema, la Organización Mundial de la Salud (OMS) y Organización de las Naciones Unidas (ONU), han incluido en sus programas líneas estratégicas que contemplan la Salud Sexual y Reproductiva (3).

Una de las características de la población adolescente que los conllevan a diferentes tipos de problemas, en especial cuando existe desconocimiento de como ejercer su sexualidad, es cuando deben adquirir compromisos a temprana edad, reflejándose en la falta de oportunidades sobre todo para la madre joven, debido a que debe interrumpir su proceso formativo, trayendo en algunas ocasiones consecuencias definitivas para su 
futuro. Una de estas consecuencias es el aborto, puesto que en los últimos años la tasa se ha incrementado, dando como resultado una mayor morbi-mortalidad materno-infantil (4).

En Colombia la política nacional de salud sexual y reproductiva (SSR), busca reducir el embarazo en adolescente, al mismo tiempo prevenir y brindar atención a las Infecciones de Transmisión Sexual (ITS), en especial al VIH y el SIDA (5).Según datos de la OMS, se estimó aproximadamente 340 millones de casos nuevos de ETS curables a nivel mundial, $80 \%$ de estos en países en desarrollo, América Latina y el Caribe ocupan el tercer lugar de frecuencia con incidencia de 38 millones de casos, preocupando la relación ETS y VIH (6). Reportes de ONU-SIDA, para América Latina y el Caribe en el año 2011, notificó que 83000 personas contrajeron el VIH (7) y la Encuesta Nacional de Demografía y Salud (ENDS) del 2010 señaló que en Colombia existe subregistro, estimando aproximadamente 500000 personas viviendo con VIH (8).

Hoy día, la mayoría de los adolescentes han tenido experiencias sexuales y muchos de ellos de manera regular, sin embargo son pocos los que poseen información de cómo protegerse de las enfermedades de transmisión sexual y de los riesgos que corren al enfermarse. Por lo tanto, es necesaria su conformación como sujetos de derechos en la práctica y no sólo objetos de derechos en la legislación, dado que se encuentran en una etapa de plena formación cívica (9).

Desde esta perspectiva, los derechos humanos han sido declarados como la principal arma de defensa contra cualquier transgresión económica, social, política y cultural. Es así, que los derechos sexuales y reproductivos forman parte de los derechos humanos, ya que protegen la capacidad del individuo para decidir de forma autónoma lo que desea hacer con su cuerpo y con su vida sin afectar su salud. De esta manera, el objetivo de este estudio es determinar que conocimientos sobre salud sexual y reproductiva poseen las estudiantes del primer semestre de Enfermería de una Universidad de Barranquilla.

En América Latina son innumerables las cifras que describen claramente la situación sexual y reproductiva de este continente entre las que cabe mencionar las señaladas por la Red de Salud de las Mujeres Latinoamericanas y del Caribe (2003) “...cada minuto se embarazan 380 mujeres y 190 de esas gestaciones son indeseadas... Las complicaciones del embarazo y el parto son causa principal de defunción y discapacidad para mujeres de 15 a 49 años de edad."(10).

Investigaciones realizadas en jóvenes universitarios, arrojan que una parte de los encuestados señalan hacer uso del preservativo con el fin de evitar embarazos, demostrándose la falta de conocimiento en el uso del mismo para evitar contraer una ITS o SIDA (11). De este modo, otro estudio realizado en estudiantes españoles sobre la percepción de riesgo de la transmisión heterosexual, demuestra que el menor riesgo percibido es asociado a una actividad sexual con una pareja afectiva (12). En tanto, Flores y Leyva (13), observan en representaciones de adolescentes, que la utilidad del condón se relaciona más con la prevención de embarazos, que con la prevención de la ITS. De esta manera, el desconocimiento de prevalencia de las infecciones de transmisión sexual, es uno de los aspectos más preocupantes para su abordaje, debido a que se deben tomar medidas estrictamente necesarias para prevenir complicaciones que agraven la salud del individuo $(14,15)$.

Es de notar que la sexualidad en los jóvenes es fundamental debido a que en esta etapa se desarrolla muchos componentes de la vida adulta. Los adolescentes deben hacer parte de estructuras sociales, y es el ambiente universitario donde influye la construcción de ese rol, facilitando la toma de decisiones en los comportamientos de su vida sexual (16-19).

\section{ATERIALESY MÉTODOS}

Estudio de carácter cuali-cuantitativo, descriptivo no experimental, de corte transversal. La población estuvo compuesta por estudiantes universitarios de primer semestre de enfermería matriculados en una universidad de Barranquilla, Colombia, en el primer período de 2015.En el estudio participaron 90 estudiantes que cumplieron con los criterios de inclusión.

Se diseñó un instrumento, el cual fue validado por investigadores del grupo de cuidado de la Universidad Simón Bolívar. Para calcular la confiabilidad del instrumento se empleó SPSS for Windows, utilizando el coeficiente KR-20 (20), cuyo resultado fue 0,82 que indicó muy alta confiabilidad. Seguido se realizó una charla explicativa del estudio a realizar, a lo que posterior cada estudiante firmó el consentimiento informado y diligenció la encuesta de Salud Sexual y 
Reproductiva, manteniendo la confidencialidad de los datos.

Los resultados fueron tabulados, organizados, interpretados y analizados por frecuencia absoluta (fa) y porcentual (\%), representados en gráficos y tablas con sus respectivos análisis de tipo descriptivo.

\section{RESULTADOS}

La población estuvo compuesta por 120 estudiantes de primer semestre de enfermería matriculados en el primer semestre académico de 2015. Se aplicaron 90 encuestas excluyendo el $25 \%$ por no cumplir los estudiantes con la edad requerida.

\section{Características demográficas}

En la tabla 1 se evidencia que el $84,45 \%$ de la totalidad poblacional son de sexo femenino, con un rango de edad del $81,11 \%$ menor de 20 años, predominando que el $92,23 \%$ se encuentra soltero, siendo esta diferencia estadísticamente significativa.

Tabla 1. Características demográficas de los estudiantes de primer semestre de enfermería matriculados en el primer semestre de 2015. Encuesta Salud Sexual y Reproductiva, Barranquilla, Colombia.

\begin{tabular}{ccc}
\hline Variable & Niveles & $n(\%)$ \\
\hline \multirow{2}{*}{ Sexo } & Femenino & $76(84 \%)$ \\
& Masculino & $14(16 \%)$ \\
\hline \multirow{2}{*}{ Edad } & $\leq 20$ años & $73(81 \%)$ \\
& $>20$ años & $17(19 \%)$ \\
\hline \multirow{2}{*}{ Estado civil } & Soltero & $83(92 \%)$ \\
& Casado & $3(3 \%)$ \\
& Unión libre & $3(3 \%)$ \\
Religión & Viuda (o) & $1(1 \%)$ \\
\hline \multirow{2}{*}{ Nivel Educativo } & Católica & $57(63 \%)$ \\
& Protestante & $32(36 \%)$ \\
\hline \multirow{2}{*}{ Nivel Socio- } & Ninguna & $1(1 \%)$ \\
económico & Secundaria & $72(80 \%)$ \\
& Técnico & $18(20 \%)$ \\
\hline \multirow{2}{*}{ Zona } & Bajo & $27(30 \%)$ \\
& M edio & $58(64 \%)$ \\
\hline [Fuente: Resultado del Instrumento aplicado] & $5(6 \%)$ \\
\hline
\end{tabular}

En relación a la religión que profesa el 63,33\% siguen el catolicismo, el $80 \%$ son bachilleres, de los cuales el $64,44 \%$ son de nivel socioeconómico medio. Donde el $82,22 \%$ de la población procedía de la zona urbana.

Identificación de la actividad sexual

Se encontró que el $62,22 \%$ de los estudiantes habían iniciado su vida sexual, con un promedio del $46,67 \%$ en rango de edad entre los 16 y 19 años. Identificando que el $45,55 \%$ tienen un compañero sexual permanente.

Tabla 2. Identificación de inicio de actividad sexual, compañeros y frecuencia de relaciones sexuales.

\begin{tabular}{|c|c|c|}
\hline Variable & Niveles & $n(\%)$ \\
\hline \multirow{2}{*}{$\begin{array}{l}\text { Ha iniciado su } \\
\text { actividad sexual }\end{array}$} & $\mathrm{SI}$ & $56(62 \%)$ \\
\hline & NO & $34(38 \%)$ \\
\hline \multirow{4}{*}{$\begin{array}{l}\text { En qué edad } \\
\text { inicio su } \\
\text { actividad sexual }\end{array}$} & Antes de los 15 años & $12(13 \%)$ \\
\hline & Entre 16 y 19 años & $42(47 \%)$ \\
\hline & Mayor de 20 años & $3(3 \%)$ \\
\hline & No ha iniciado actividad sexual & $33(37 \%)$ \\
\hline \multirow{3}{*}{$\begin{array}{l}\text { Estabilidad de } \\
\text { compañero } \\
\text { sexual }\end{array}$} & Permanente & 41 (46\%) \\
\hline & Ocasional & $16(18 \%)$ \\
\hline & No ha iniciado actividad sexual & $33(37 \%)$ \\
\hline \multirow{4}{*}{$\begin{array}{l}\text { Identificación de } \\
\text { compañeros (a) } \\
\text { sexuales }\end{array}$} & 0 a 1 & $30(33 \%)$ \\
\hline & 2 a 5 & $18(20 \%)$ \\
\hline & Mayor de 5 & $9(10 \%)$ \\
\hline & No ha iniciado actividad sexual & $33(37 \%)$ \\
\hline \multirow{4}{*}{$\begin{array}{l}\text { Frecuencia de la } \\
\text { actividad sexual }\end{array}$} & No relaciones sexuales & $7(8 \%)$ \\
\hline & De 1 a 3 relaciones por semana & $43(48 \%)$ \\
\hline & $\begin{array}{l}\text { De } 4 \text { o más relaciones por } \\
\text { semana }\end{array}$ & $7(8 \%)$ \\
\hline & No ha iniciado actividad sexual & $33(37 \%)$ \\
\hline
\end{tabular}

Respecto a los estudiantes el $36,67 \%$ no han iniciado su actividad sexual, mientras que el $33,33 \%$ han tenido un solo compañero sexual, teniendo el $47,77 \%$ relaciones de 1 a 3 veces por semana (Tabla 2).

\section{Identificación de los métodos anticonceptivos}

En cuanto la utilización de métodos anticonceptivos el $53,33 \%$ coincide en la no utilización, destacando que hay que tener en cuenta que el $36,67 \%$ no ha iniciado su vida sexual, por lo que el $30 \%$ emplea el condón como medio de protección a las enfermedades de transmisión sexual y embarazos no deseados (Tabla 3). 
Tabla 3. Conocimientos y utilización de los métodos de planificación familiar.

\begin{tabular}{lll}
\hline Variable & Niveles & $\mathrm{n}(\%)$ \\
\hline Utiliza algún método & $\mathrm{Si}$ & $42(47 \%)$ \\
anticonceptivo & No & $48(53 \%)$ \\
& Ninguno & $40(44 \%)$ \\
& Condón & $27(30 \%)$ \\
& Anticonceptivo oral & $9(10 \%)$ \\
& Anticonceptivo & \\
& inyectable & $12(13 \%)$ \\
Qué tipo de método do & Dispositivo intrauterino & \\
anticonceptivo utiliza & Ligadura o Vasectomía & $1(1 \%)$ \\
\hline & Siempre & $25(28 \%)$ \\
\cline { 2 - 3 } Frecuencia de & Casi siempre & $8(9 \%)$ \\
utilización del & Algunas veces & $12(13 \%)$ \\
preservativo & Nunca & $45(50 \%)$ \\
\hline & Iniciativa propia & $(0 \%)$ \\
Que aspectos & Mi compañera (o) & $42(47 \%)$ \\
influyeron en la & Amigos & $4(4 \%)$ \\
decisión de planificar o 0 & Familiares & $1(1 \%)$ \\
tomar métodos & No responal de salud & $3(3 \%)$ \\
anticonceptivos & No Datos & $2(2 \%)$ \\
\hline Asiste a programa de & Si & $38(42 \%)$ \\
planificación familiar & No & $8(9 \%)$ \\
\hline Posee conocimientos & Si & $82(91 \%)$ \\
sobre anticoncepción & No & $77(86 \%)$ \\
\hline [Fuente: Instrumento aplicado] & $13(14 \%)$ \\
\hline
\end{tabular}

Con respecto a la frecuencia de utilización del preservativo el $27,77 \%$ afirma utilizarlo siempre, reafirmando un $46,67 \%$ haber tomado la decisión de planificar por iniciativa propia, donde solo el 8,89\% asisten a programa de planificación familiar, pero el $85,55 \%$ señalan poseer conocimientos sobre anticoncepción.

Identificación de infecciones sobre transmisión sexual

El $100 \%$ de los y las adolescentes conocían sobre las infecciones de transmisión sexual, el $94,45 \%$ no ha padecido ningún tipo de ITS, y el 73,34\%nunca se ha compartir agujas, ni se ha colocado piercing, ni realizado tatuajes. Mientras que el $80 \%$ no se ha realizado la prueba de VIH. (Tabla 4).

Tabla 4. Identificación de infecciones sobre transmisión sexual

\begin{tabular}{lll}
\hline Variable & Niveles & $\mathrm{n}(\%)$ \\
\hline $\begin{array}{l}\text { Conoce sobre las } \\
\text { infecciones de transmisión } \\
\text { sexual }\end{array}$ & $\mathrm{Si}$ & $(0 \%)$ \\
\hline Ha presentado infección de & $\mathrm{Si}$ & $90(100 \%)$ \\
transmisión sexual & $\mathrm{No}$ & $5(6 \%)$ \\
\hline \multirow{2}{*}{$\begin{array}{l}\text { Se ha realizado alguno de } \\
\text { estos procedimientos }\end{array}$} & Compartir agujas & $0(0 \%)$ \\
& Piercing & $14(16 \%)$ \\
& Tatuajes & $10(11 \%)$ \\
\hline
\end{tabular}

\begin{tabular}{lll}
\hline Variable & Niveles & $\mathrm{n}(\%)$ \\
\hline Se ha realizado prueba de & $\mathrm{Si}$ & $18(20 \%)$ \\
\cline { 2 - 3 } VIH & No & $72(80 \%)$ \\
\hline \hline [Fuente: Instrumento aplicado] &
\end{tabular}

Identificación de embarazos

En la tabla 5 , se evidencia que el $10 \%$ de las adolescentes han estado embarazadas, reflejando que el $5,55 \%$ no planeo estarlo, mientras que el $93,33 \%$ no se ha practicado ninguna clase de abortos, a lo que el $4,45 \%$ afirman que ha presentado aborto espontaneo.

Tabla 5. Relación de embarazos y práctica de abortos

\begin{tabular}{lll}
\hline Variable & Niveles & $\mathrm{n}(\%)$ \\
\hline \multirow{2}{*}{ Ha estado o está embarazada } & $\mathrm{Si}$ & $9(10 \%)$ \\
& $\mathrm{No}$ & $81(90 \%)$ \\
\hline \multirow{2}{*}{ El embarazo fue planeado } & $\mathrm{Si}$ & $4(4 \%)$ \\
& $\mathrm{No}$ & $5(6 \%)$ \\
\hline \multirow{2}{*}{ Ha presentado aborto } & Ninguno & $81(90 \%)$ \\
\hline \multirow{2}{*}{ Tipo de aborto } & $\mathrm{Si}$ & $6(7 \%)$ \\
& No & $84(93 \%)$ \\
& Espontaneo & $4(4 \%)$ \\
& Inducido & $2(2 \%)$ \\
\hline
\end{tabular}

[Fuente: Instrumento aplicado]

Identificación de programa de salud sexual y reproductiva

La población estudiada considera en un $96,67 \%$ que la universidad tenga un programa vigente de salud sexual y reproductiva, donde el $35,56 \%$ le gustaría que utilizaran talleres para acceder a la información (Tabla 6).

Tabla 6.Acceso a programa de salud sexual y reproductiva

\begin{tabular}{lll}
\hline Variable & Niveles & $\mathrm{n}(\%)$ \\
\hline $\begin{array}{l}\text { Considera necesario que la } \\
\text { universidad tuviera un } \\
\text { programa de salud sexual y }\end{array}$ & $\mathrm{Si}$ & $87(97 \%)$ \\
reproductiva & $\mathrm{No}$ & $3(3 \%)$ \\
\hline & $\begin{array}{l}\text { Currículo de } \\
\text { estudio }\end{array}$ & $(0 \%)$ \\
$\begin{array}{l}\text { Que medio le gustaría } \\
\text { utilizar para acceder al } \\
\text { programa de salud sexual y y } \\
\text { reproductiva }\end{array}$ & $\begin{array}{l}\text { Talletos } \\
\text { Personas a cargo } \\
\text { del programa }\end{array}$ & $32(36 \%)$ \\
& $\begin{array}{l}\text { Página web } \\
\text { institucional }\end{array}$ & $26(29 \%)$ \\
\hline $\begin{array}{ll}\text { [Fuente: Instrumento aplicado] } \\
\text { DISCUSIÓN }\end{array}$ & & \\
En los últimos años la salud sexual y reproductiva se
\end{tabular}


ha convertido en un tema de gran interés a nivel mundial (21). Según datos aportados por UNICEF, en América Latina y el Caribe más del $50 \%$ de los jóvenes menores de 17 años han iniciado su vida sexual. Un estudio realizado por Valenzuela, advierte que el $66 \%$ de hombres y el $60 \%$ de mujeres, se han iniciado sexualmente entre los 15 y 18 años (22).

El inicio cada vez más temprano de las relaciones sexuales, van acompañadas en gran parte bajo a algunos efectos de sustancias como el alcohol o las drogas, así como la falta de conocimiento sobre el buen manejo de su sexualidad. Esto indica que a pesar del acceso a la información que se muestra por diferentes medios, los jóvenes aún no está asimilando todo lo referente a la salud sexual y reproductiva, cuestionando la calidad con la que los organismos académicos y de salud están impartiendo la educación, convirtiendo a la juventud en un grupo poblacional vulnerable frente a situaciones que ponen en riesgo su salud.

Estudios realizados en Colombia por Mosquera (2003) y Urrea (2006) encontraron que la población joven tenía conocimientos deficientes sobre salud sexual y reproductiva, que los llevaría a tomar conductas de riesgo $(23,17)$. De igual manera un estudio realizado en china, reportó que la población entre más temprano inicie la vida sexual, incurre en mayores conductas de riesgo con mayores tasas de embarazos no deseados, y adquisición de ETS o VIH (24). De esta forma este estudio contribuye al fortalecimiento de las directrices mundiales, nacionales y departamentales que al respecto dictan los estamentos de vigilancia sobre los derechos sexuales y reproductivos en las personas más vulnerables.

Otras investigaciones en estudiantes universitarios toman como referentes estratégicos en la prevención de enfermedades de transmisión sexual y embarazo no deseado, el uso del condón en sus prácticas e interacciones sociales (25). Así mismo, encontraron una población universitaria joven ( $\leq 20$ años), proveniente de una zona urbana predominantemente heterosexual y con conocimientos previos referente a Salud Sexual y Reproductiva, afirmando que en la escuela es donde se obtiene la primera fuente de información (26).De esta manera, uno de los alcances de esta investigación seria el establecimiento de un programa de educación para la población universitaria, que sirva de referencia para otros centros educativos del Caribe y de Latinoamérica.
Lo que se pretende es encaminar estos programa si la prevención de las enfermedades de transmisión sexual y embarazo no deseado en adolescentes, y que estos sean liderados universidades en conjunto con las Secretarias de Salud y Educación del departamento.

\section{AGRADECIM IENTOS}

A la Universidad Simón Bolívar de Barranquilla por su apoyo con el recurso humano; a los estudiantes Ludís Barros, Katy Castro, Luisa Marpia, Ashley Núñez, Angie Polo quienes contribuyeron en la aplicación de las encuestas, recolección y análisis de datos del estudio que origina este artículo.

\section{CONFLICTO DE INTERESES}

El autor manifiesta no tener ningún conflicto.

\section{REFERENCIAS BIBLIOGRÁFICA}

1. World Health Organization (WHO). Reproductive Health.WHO; 2013.[Internet]. Disponible en: http://www.who.int/topics/reproductive_health/en/

2. United Nations. International Conference on population and Development (ICPD), Summary of the Programme of Action. 1994. [Internet]. Disponible en: http://www.un.org/ecosocdev/geninfo/populatin/ic pd.htm\#intro.

3. UNFPA. UNFPA, Colombia. Colombia; 2013. [Internet]. Disponible en:http://www.unfpa. org.co/menuSup.php?id=1.

4. IGLESIAS CORTIT, L. Contracepción en la adolescencia. Rev. Iberoamericana de Fertilidad.Vol XIV, no 1, 3-8, Enero-Febrero, 1997.

5. Ministerio de la Protección Social. Dirección General de Salud Pública. Políticanacionaldesalud sexual y reproductiva; 2003.

6. World Health Organization (WHO). Global prevalence and incidence of selected curable Sexually Transmitted Infections overview and estimates.Geneve: WHO; 2001.

7. ONUSIDA. Hoja Informativa Regional 2012, ONUSIDA. ONUSIDA; 2012. [Internet]. Disponible en: http:// Www.uniaids.org/ en/media/uniaids/contentassets/documents/epidem iology/2012/gr2012/2012_FS_regional_la_caribbean es.pdf. Consultado mayo $20 \overline{15}$.

8. PROFAMILIA. Encuesta Nacional de demografía y salud (ENDS): Salud Sexual y Reproductiva en Colombia. Colombia, PROFAM ILIA; 2010. [Internet]. Disponible en:http://www.profamilia.org.co/encuestas/Profamil 
ia/Profamilia/index. php? option=com_content\&view $=$ article\&id $=149 \&$ Itemid $=119$.

9. CABRERA DEL CASTILLO, Mạ. A., MARTÍN DÍAZ, J. LUQUÍN AJURIA, A. ORUETA SÁNCHEZ, R., LÓPEZ DE CASTRO, F. Mayo, 1996 Conocimientos, actitudes y comportamientos sobre sexualidad en dos colectivos de jóvenes-adolescentes.

10. RED DE SALUD DE LAS MUJERES LATINOAM ERICANAS Y DEL CARIBE (2003) Situación Sexual $Y$ Reproductiva Documento en línea. Disponible en: www.reddesalud.org/espanol/.

11. Vásquez ML, Argote LA, Castillo E, Cabrera MJ, Gonzales D, M ejía ME, Villaquirán ME. Apropiación de los derechos sexuales y reproductivos en adolescentes: una experiencia desde la teoría de la acción razonada. Colombia M édica. 2005; 36 (1): 1424.

12. Lameiras M, Rodríguez Y, Dafonte S. Evolución de la percepción de riesgo de la transmisión heterosexual del VIH en universitarios/as españoles/as. Universidad de Oviedo. Ovie-do España. Psicothema. 2002; 14 (2): 255 -261.

13. Triguero MC, Bergamo de Oliveira A, Rodríguez C, Borges Da Silva G. De la representación a la práctica sexual. Un estudio exploratorio de las representaciones socia les so-bre las enfermedades sexualmente transmisibles. Revista Intercontinental de psicología y educación. 2006; 8 (2): 79-30.

14. Wein K, Kavoussi L, Novick A, Peters G. Urología. 9a edición. Panamericana. 2008; 371-401.

15. Lartigue $T$, Ávila $H$. Sexualidad y Reproducción Humana en M éxico. Plaza Valdés. 1996; 4: 143-162.

16. Montero VA. Educación sexual: un pilar fundamental en la sexualidad de la adolescencia.RevMed Chile 2011; 139:1249-52.

17. Urrea GF, Congolino ML, Herrera HD, Reyes JI, Botero WF. Comportamientos sexuales e incidencia de los programas de salud sexual y reproductiva en estudiantes de secundaria de sectores populares y de la universidad pública en la ciudad de Cali, Colombia. CadSaúde Pública 2006; 22(1):209-15.

18. Serrano GI, Cintrón BF, Rodriguez Y, Acosta PE, WaltersPK. Una mirada a la sexualidaddesde el Caribe:Implicaciones de su estudio para las Ciencias Sociales. Revista deCiencias Sociales 2005; 14:10-21.

19. Sosa SI. La juventud y su contexto. En: Significados de la salud y la sexualidad de jóvenes. 1ra ed, México: Instituto Nacional de las Mujeres (INM UJERES); 2005.

20. Morales Vallejo P. Estadística aplicada a las ciencias sociales. Madrid: Universidad Pontifica Comillas; 2011.

21. Organización Mundial de la Salud (OMS). Estrategia Mundial de Prevención y Control de las Infecciones de Transmisión Sexual 2006-2015. OMS; 2007. Report: WC142.

22. Valenzuela Rivera Ester, Casas Becerra Lidia. DERECHOS SEXUALES $Y$ REPRODUCTIVOS: CONFIDENCIALIDAD Y VIH/SIDA EN ADOLESCENTES CHILENOS. Acta bioeth. [revista en la Internet]. 2007 Disponible en: http://www.scielo.cl/scielo.php?script=sci_arttext\&p id $=S 1726569 \times 2007000200008 \&$ lng $=e s$. http://dx.doi.org/10.4067/S1726569X2007000200008

23. M osquera J., Mateus (C. Conocimientos, actitudes y prácticas sobre métodos de planificación familiar, VIH-SIDA y el uso de los medios de comunicación en jóvenes. Colombia M édica 2003; 34(4):206-12.

24. Ma Q, Ono KM, Cong L, Xu G, Pan X, Zamani S, et al. Early initiation of sexual activity: a risk factor for sexually transmitted diseases, HIV infection, and unwanted pregnancy among university students in China. BioM ed Central 2009; 9(111):1-8.

25. Ballester R. Niños y jóvenes en el Norte de Nicaragua: análisis epidemiológico de las prioridades psico-socio-sanitarias para una intervención psicosociosanitaria. Castellón: Publicaciones de la Universitat aume l; 2005.

26. United Nations Statistics Division. Population size and density.New York, United Nations; 2011.[Internet]. Disponible en: http://unstats.un.org/unsd/demographic/sconcerns/ popsize/size2.htm\#WM . • 